\title{
Evidence from bioinformatics, expression and inhibition studies of phosphoinositide-3 kinase signalling in Giardia intestinalis Siân SE Cox ${ }^{1}$, Mark van der Giezen ${ }^{2}$, Sarah J Tarr ${ }^{1}$, Mark R Crompton ${ }^{1}$ and Jorge Tovar*1
}

Address: ${ }^{1}$ School of Biological Sciences, Royal Holloway University of London, Egham, Surrey, UK and ${ }^{2}$ School of Biological and Chemical Sciences, Queen Mary, University of London, Mile End Road, London, UK

Email: Siân SE Cox - s.s.e.cox@rhul.ac.uk; Mark van der Giezen - m.vandergiezen@qmul.ac.uk; Sarah J Tarr - s.tarr@rhul.ac.uk; Mark R Crompton - m.crompton@rhul.ac.uk; Jorge Tovar* - j.tovar@rhul.ac.uk

* Corresponding author

Published: 18 May 2006

BMC Microbiology 2006, 6:45 doi:10.1186/147/-2180-6-45
Received: 03 February 2006

Accepted: 18 May 2006

This article is available from: http://www.biomedcentral.com/I47/-2/80/6/45

(c) 2006 Cox et al; licensee BioMed Central Ltd.

This is an Open Access article distributed under the terms of the Creative Commons Attribution License (http://creativecommons.org/licenses/by/2.0), which permits unrestricted use, distribution, and reproduction in any medium, provided the original work is properly cited.

\begin{abstract}
Background: Giardia intestinalis is a parasitic protozoan and major cause of diarrhoeal disease. Disease transmission is dependent on the ability of the parasite to differentiate back and forth between an intestine-colonising trophozoite and an environmentally-resistant infective cyst. Our current understanding of the intracellular signalling mechanisms that regulate parasite replication and differentiation is limited, yet such information could suggest new methods of disease control. Phosphoinositide-3 kinase (PI3K) signalling pathways have a central involvement in many vital eukaryotic processes, such as regulation of cell growth, intracellular membrane trafficking and cell motility. Here we present evidence for the existence of functional PI3K intracellular signalling pathways in G. intestinalis.
\end{abstract}

Results: We have identified and characterised two genes, Gipi3k I and Gipi3k2, which encode putative PI3Ks. Both genes are expressed in trophozoites and encysting cells, suggesting a possible role of GiPI3KI and GiPI3K2 in regulating giardial growth and differentiation. Extensive nucleotide and amino acid sequence characterisation predicts that both encoded $\mathrm{PI} 3 \mathrm{~K} s$ are functional as indicated by the presence of highly conserved structural domains and essential catalytic residues. The inhibitory effect of the PI3K inhibitor LY294002 on trophozoite proliferation also supports their functionality. Phylogenetic analysis supports the identity of GiPI3KI as a Class I isoform and GiPI3K2 as a Class III isoform. In addition, giardial genes encoding putative homologues of phosphoinositide-metabolising enzymes such as PTEN, MTM, PIPkin and PI 5-phosphatase as well as downstream effectors with phosphoinositide-binding domains have been identified, placing GiPI3KI and GiPI3K2 in a broader signalling context. Compared with twenty-six PI3Ks from other organisms, GiPI3K I and GiPI3K2 are unique in that they contain large insertions within their highly conserved kinase domains. The function of these insertions is unknown; however we show here that they are not intron-derived and would probably not hinder substrate binding. These insertions may represent a plausible drug target.

Conclusion: $G$. intestinalis encodes and expresses two putative PI3Ks, at least one of which appears to be required during normal parasite proliferation. The identification of Class I and Class III but not Class II isoforms suggests that both extracellularly-initiated signalling (Class I-regulated) and intracellular vesicle trafficking (Class III-regulated) might be controlled by PI3Ks in $G$. intestinalis. The presence of genes encoding putative homologues of phosphoinositidemetabolising enzymes and downstream effectors in the $G$. intestinalis genome further suggests that the overall architecture of PI3K signalling may be comparable with pathways present in other better-studied organisms. 


\section{Background}

The parasitic protozoan Giardia intestinalis (syn. lamblia, duodenalis) is a common cause of water-borne diarrhoeal disease, particularly in the developing world. G. intestinalis's infective ingenuity stems from its ability to switch between two morphologically distinct states: an externally protected, inert cyst and a flagellated colonizing trophozoite that can attach to host cells in the small intestine. This attachment leads to the unpleasant symptoms associated with giardiasis [1]. Differentiation of G. intestinalis has been studied extensively in terms of analysing the morphological events associated with encystation (trophozoite-to-cyst) and excystation (cyst-to-trophozoite) [1], yet little is known about the molecular circuitry that regulates differentiation. Although limited in their number, a few studies have given some crucial insights into the intracellular signalling pathways that may allow the organism to respond to environmental cues. For instance, protein kinase A and cyclic-AMP, in addition to calcium and calmodulin have been shown to play an important role in trophozoite motility and initiation of excystation [2-4] while catalytically active homologs of the intracellular signalling mitogen-activated protein kinase family in G. intestinalis appear to be important for growth and encystation [5]. The potential diversity of intracellular signalling mechanisms in $G$. intestinalis is illustrated by the recent characterisation of a giardial protein kinase B-like molecule [6] and by the demonstration that cholesterol starvation in vitro induces Giardia encystation [7]. The latter suggests a role for lipids and lipid signalling in parasite differentiation.

Phosphatidylinositol (PtdIns) is a basic building block for intracellular inositol lipids in eukaryotic cells and is present in trace amounts in G. intestinalis trophozoites and encysting cells cultured in vitro [8]. It has been shown that $G$. intestinalis uses inositol headgroup exchange enzymes to convert free inositol to PtdIns [9]. In Dictyostelium discoideum, yeast and mammalian cells, PtdIns can be reversibly phosphorylated at the D-3 position of its inositol ring by phosphoinositide 3-kinases (PI3Ks), and at other positions by distinct kinases, to form phosphorylated PtdIns; PtdIns and its phosphorylated derivatives are collectively referred to as phosphoinositides (PIs) [10]. Although PIs constitute a minor fraction of total cellular lipids in eukaryotic cells, they are biologically important as second messengers and their levels must be accurately regulated [11].

Although PtdIns has been detected in Giardia [8] it is unclear whether this parasite utilises PI3K-mediated intracellular signalling mechanisms to regulate its growth and differentiation. PI3K signalling is associated with many eukaryotic events such as regulation of cell growth, intracellular membrane trafficking and cell motility [12], each of which is important in the lifecycle of G. intestinalis. PI3K signalling has been extensively characterised in mammals, yeast and most notably in the slime mold Dictyostelium discoideum, where several PI3Ks are known to be important for normal cell growth, multicellular development, aggregation and chemotaxis $[13,14]$.

PI3Ks become activated in response to numerous agonists and the resulting PIs initiate the recruitment of signalling proteins to membranes - specifically by way of PI-binding domains present on the proteins. In this way, membrane signals can be spatially restricted and highly controlled. A well-characterised example of a PI-binding signalling protein is protein kinase $\mathrm{B}(\mathrm{PKB})$, which, when membranerecruited by $\mathrm{PI}$, is only then able to phosphorylate specific substrates in order to regulate apoptosis, protein synthesis, cell growth and cell cycling [12]. To date, eight mammalian PI3Ks have been identified and they comprise three classes of isozymes on the basis of sequence similarity and substrate preference in vivo [11]. Class I PI3Ks phosphorylate PtdIns, PtdIns(4)P and PtdIns $(4,5) \mathrm{P}_{2}$, Class II PI3Ks phosphorylate PtdIns and PtdIns(4)P but not PtdIns $(4,5) \mathrm{P}_{2}$ and Class III PI3Ks (sometimes referred to as Vps34, after the isoform detected in yeast [15]) exclusively phosphorylate PtdIns. All PI3Ks characterised to date have a multi-domain structure, comprising a highly conserved two-lobed catalytic kinase domain at their Cterminus, a PIK domain which acts as a scaffold around which the enzyme is folded and a C2 or C2-like domain that in other proteins binds phospholipids and so may be involved in substrate targeting. The PI3Ks also have domains that mediate enzyme activation and promote enzyme interaction with the plasma membrane, namely the Ras Binding Domains (RBDs) common to Class I and II isoforms, and adaptor protein binding domains [11].

Using mammalian and yeast PI3K protein sequences as queries, we searched the G. intestinalis genome [16] to identify putative molecular homologues. Here, we report on the characterisation of two giardial genes, which we term Gipi3k1 and Gipi3k2, encoding putative PI3Ks. The presence of well conserved structural PI3K domains in these sequences and the detection of specific transcripts for both genes in trophozoites and encysting cells suggest that GiPI3K1 and GiPI3K2 are functional enzymes. Functionality of at least one of the putative PI3Ks is experimentally demonstrated by the specific and significant inhibitory effect of a PI3K inhibitor on trophozoite proliferation. We further substantiate the existence of PI3K signalling in $G$. intestinalis by identifying additional homologs which may be important for the metabolism and downstream effects of the giardial PI3K enzymatic products. 
Table I: Analysis of the giardial PI3K domains. Percentage identity values for the full-length and domain-only sequences of GiPI3K I and GiPI3K2 with other PI3K isoforms following their sequence alignment.

\begin{tabular}{|c|c|c|}
\hline & GiPI3KI & GiPI3K2 \\
\hline Full- length & 39\% to Dictyostelium discoideum PIKI, a Class I PI3K & $34 \%$ to Glycine max Vps34-like (Class III) PI3K \\
\hline Ras Binding Domain (RBD) & $41 \%$ to Homo sapiens Class IB p I IO $\mathrm{P}$ PI3K & Absent \\
\hline PIK & $28 \%$ to $H$. sapiens Class IA pII $0 \alpha$ PI3K & $27 \%$ to Caenorhabditis elegans Vps34-like (Class III) PI3K \\
\hline Kinase & $39 \%$ to $D$. discoideum PIKI & $33 \%$ to Glycine max Vps34-like (Class III) PI3K \\
\hline
\end{tabular}

\section{Results \\ Identification and sequence characterisation of two putative $\mathbf{G}$. intestinalis $P I 3 K$ genes}

Using catalytic domain sequences of Class I, II and III PI3Ks as query sequences, we carried out sequence similarity searches to identify Giardia genes encoding putative PI3Ks. Our searches returned several open reading frames, two of which demonstrated similarity to PI3K genes (Table 1) and were therefore called Gipi3k1 and Gipi3k2. The predicted open reading frames of Gipi3k1 and Gipi3k2 are 6,468 bp and 4,917 bp in length respectively. Gipi3k1 is predicted to encode a 2,155 amino acid protein [GenPept: EAA41385] with a molecular mass of $242 \mathrm{kDa}$, whilst Gipi3k2 is predicted to encode a 1,638 amino acid protein [GenPept: EAA40923] with a molecular mass of $183 \mathrm{kDa}$. We independently confirmed the entire Gipi3k1 sequence given by the G. intestinalis genome project, (see Methods section) and no differences in sequence were observed.

PI3K - characteristic domains were predicted to be present and in the typical conformation for both GiPI3K1 and GiPI3K2 (Figure 1). Ubiquitin/RBD-like, C2, PIK and kinase domains were predicted for GiPI3K1 with significant expectant (E) values (i.e. the E-values were below the default threshold set for significance). C2, PIK and kinase domains were predicted for GiPI3K2, again with significant E-values. Interestingly, both putative kinase domains appeared to be interrupted by long insertions as compared with other well-studied PI3Ks (see Figure 1, dotted lines). We carried out sequence-similarity searches with the predicted Ubiquitin/RBD-like domain of GiPI3K1 which returned matches against the Ras Binding Domains of several mammalian Class I PI3Ks (see Table 1 for similarity scores). This finding is interesting, since G. intestinalis apparently contains no Ras protein-encoding sequences [17]. A recent (May 2006, unpublished) G. intestinalis genome survey by us supports this claim: we could only identify putative Rab, Ran and Rac GTPases encoded in the $G$. intestinalis genome [16], but no Rasencoding sequences. One could speculate that the putative RBD of GiPI3K1 may be recruited to membranes by another Ras-like small GTPase such as Rab.
Full-length and domain-only sequence similarity analysis of GiPI3K1 and GiPI3K2 demonstrated clear similarity with PI3Ks from other organisms, in particular GiPI3K1 is predicted to be similar to Class I PI3Ks and GiPI3K2 to Class III PI3Ks (Table 1, see also next section). Due to low conservation of sequence similarity amongst $\mathrm{C} 2$ domains from various PI3Ks, similar analysis carried out for the predicted C2 domains of GiPI3K1 and GiPI3K2 did not return significant matches to the $\mathrm{C} 2$ domains of other PI3Ks. However, C2 pairwise sequence alignments (Figure 2) demonstrated reasonable C2 domain similarity.

To further substantiate the identity of the giardial PI3K domains, pairwise alignments of the RBD of GiPI3K1 and the $\mathrm{C} 2$ and PIK domains of GiPI3K1 and GiPI3K2 were carried out with PI3Ks which shared the greatest similarity with the giardial PI3Ks (see Table 1). Figure 2 shows how the domains of GiPI3K1 and GiPI3K2 align well with domains of PI3Ks from D. discoideum and Glycine max respectively.

A similar, detailed multiple alignment analysis was carried out for the kinase domains of GiPI3K1 and GiPI3K2 against the structurally characterised kinase domain of PI3K $\gamma$ (a Class IB PI3K) from the domestic pig, Sus scrofa [18] (Figure 3). All residues required for ATP binding and kinase activity in the S. scrofa PI3K $\gamma$ are conserved in both GiPI3K1 and GiPI3K2. However, GiPI3K2 lacks one of the lysines thought to interact with the I-phosphate of PI substrate (Lys-807 in the $S$. scrofa sequence) and a lysine thought to act as a ligand for the 5-phosphate of $\mathrm{PI}(4,5) \mathrm{P}_{2}$ (Lys-973 in the S. scrofa sequence) [18]. In addition, GiPI3K2's activation loop appears to be shorter. All of these features are common to other functional Class III PI3K isoforms such as D. discoideum DdPIK5 [13], which are thought to be specific for PtdIns only since their short activation loops cannot accommodate the 4-phosphate of PtdIns(4)P substrate [18]. GiPI3K1's activation loop is similar in length to that of the S. scrofa PI3K $\gamma$. Taken together, this analysis strongly suggests that GiPI3K1 and GiPI3K2 are functional PI3Ks belonging to the Class I and Class III isoforms respectively. 


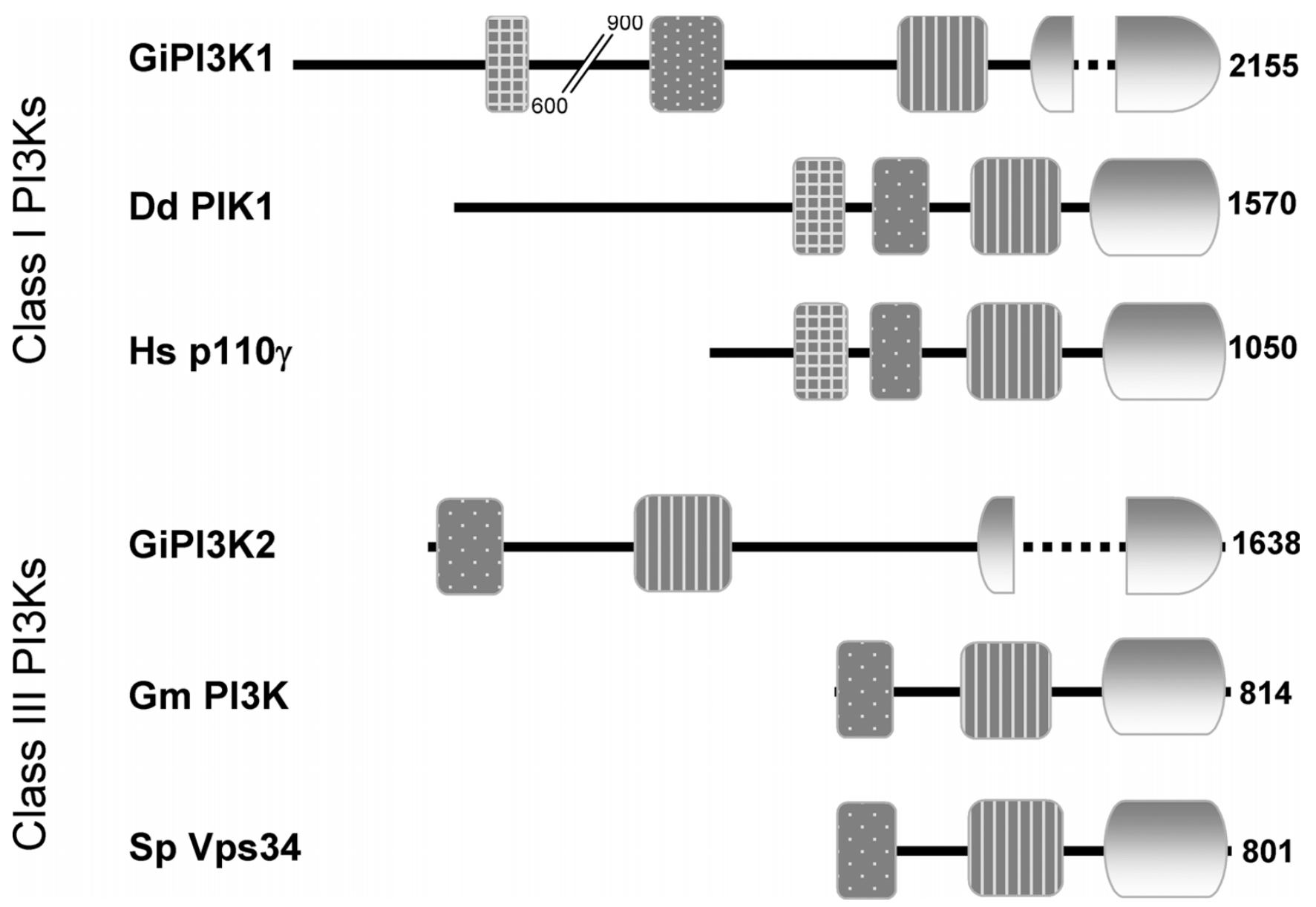

Figure I

Domain structure of GiPI3KI and GiPI3K2 compared with other PI3Ks. Top: GiPI3KI is compared to Dd PIKI, Dictyostelium discoideum Class I PI3K [GenPept: AAA8572 I] and to Hs pI I $\gamma$, Homo sapiens Class I PI3K [GenPept: NP_002640]. The GiPI3KI sequence is interrupted for the sake of clarity. Bottom: GiPI3K2 is compared to Gm PI3K, Glycine max Class III PI3K [GenPept: AAA64468] and to Sp Vps34, Schizosaccharomyces pombe Class III PI3K [GenPept: AAC49I33]. Numbers indicate amino acids. Domains are shaded as follows: checked, Ras binding; dotted, C2; striped, PIK; gradient, kinase. Apparent insertions in the giardial kinase domains are indicated by a dotted line.

Both the kinase domains of GiPI3K1 and GiPI3K2 have long insertions (96 and 235 amino acids in length respectively) that do not align with sequences in the kinase domain of S. scrofa PI3K $\gamma$, and neither do they align with each other. The positions of these insertions are intriguing since they are close to the highly conserved catalytic loop residues, and appear to sit between the $\mathrm{N}$ - and C-terminal lobes of the predicted kinase domains (Figure 3). Secondary structure predictions of the insertions indicate that they may fold into additional structures associated with the respective kinase domains. In silico three-dimensional structural sequence alignments using the structurally characterised S. scrofa PI3K $\gamma$ as a template suggest that the insertions are probably surface-exposed with respect to the two lobes of the kinase domains, aligning to a coil between two alpha helices (data not shown). Thus, these putative domains could be accommodated without preventing the correct folding of the kinase domains and may not hinder substrate access. Sequence similarity searches of the insertions did not yield any evidence in terms of predicting their biological role. Multiple alignments with twenty-six Class I and Class III PI3Ks from different organisms indicated that the apparent insertions are a unique feature of the giardial sequences. These observations prompted speculation as to the nature of the insertions. Because trophozoites undergo substantial morphological changes and alterations in gene expression [19] the insertions could be subjected to differential splicing - perhaps as a way of controlling enzyme activity [20]. However, RTPCR experiments using $36 \mathrm{hr}$ encysting cell RNA provided 


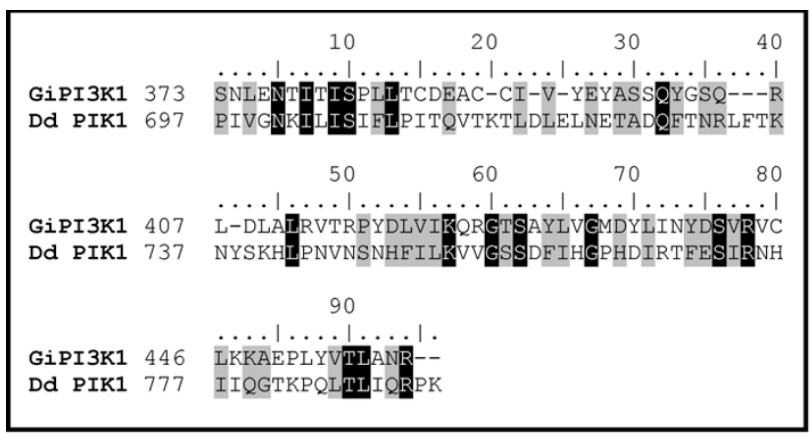

\section{a.) Ras Binding Domain}
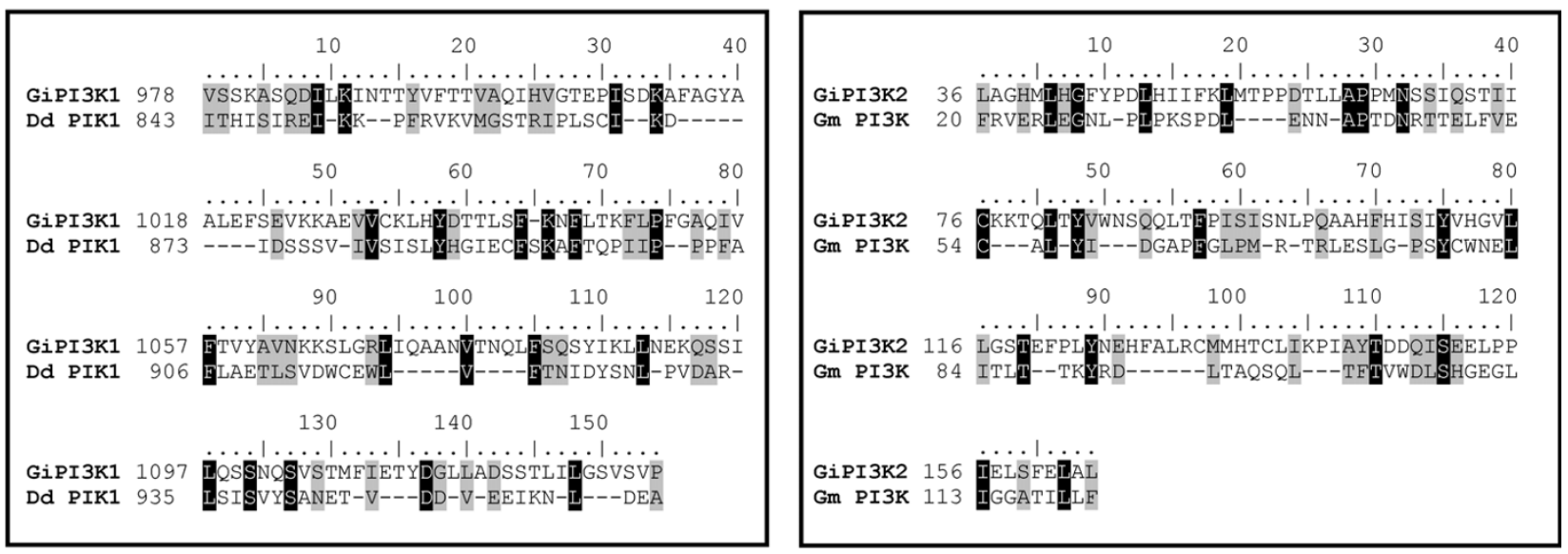

\section{b.) C2 domain}
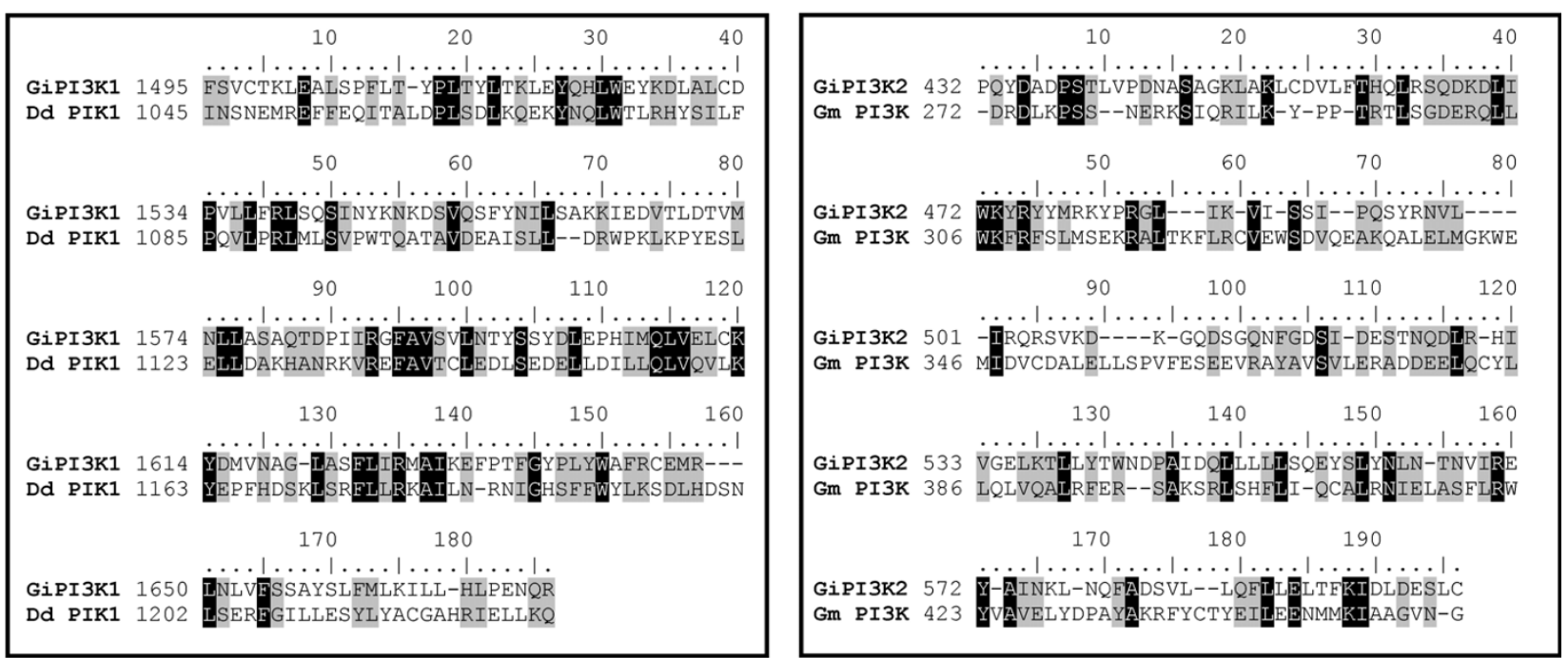

\section{c.) PIK domain}

Figure 2

PI3K domain pairwise alignments for GiPI3KI and GiPI3K2. Pairwise sequence alignment of GiPI3KI and GiPI3K2 indicates conservation in predicted Ras Binding (a), C2 (b) and PIK (c) domains. Identical aligned residues are indicated in black shading, while conserved residues are indicated by grey shading according to the PAM 250 matrix [45]. Identity/similarity percentages for the GiPI3KI alignments are $15 \% / 45 \%, 12 \% / 31 \%$ and $20 \% / 51 \%$ for the GiPI3KI RBD, C2 and PIK domains respectively, for the GiPI3K2 alignments these values are $13 \% / 31 \%$ and $16 \% / 47 \%$ for the GiPI3K2 C2 and PIK domains respectively. 


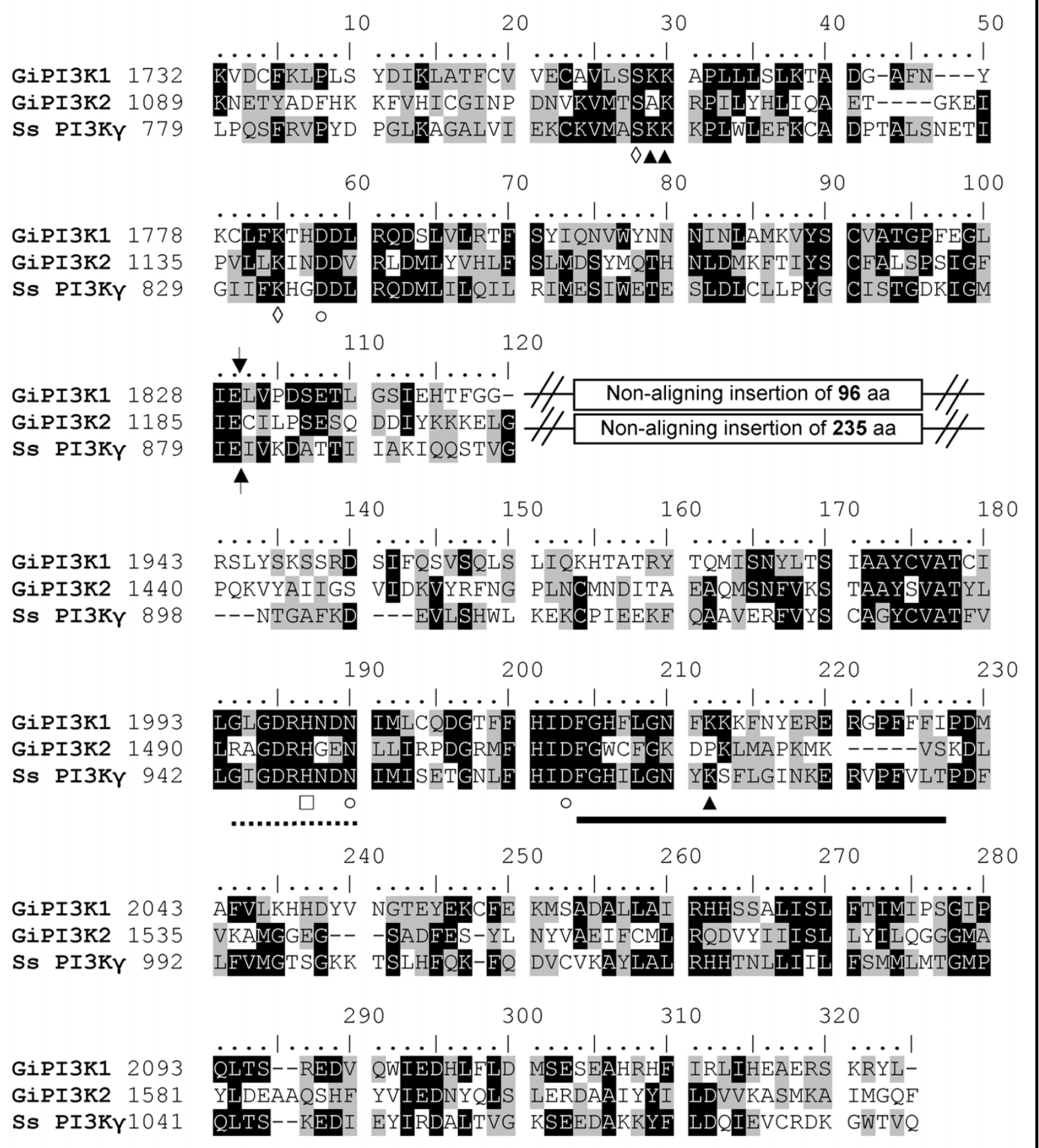

Figure 3

Detailed sequence analysis of the kinase domains of GiPI3KI and GiPI3K2. Multiple sequence alignment of the kinase domains of the giardial PI3Ks with a structurally characterised Class I PI3K-kinase domain from Sus scrofa, Ss PI3K $\gamma$, [GenPept: NP_999104] demonstrates conservation of functional residues. Sequences were aligned using ClustalW [37]. Arrows indicate the boundary between the $\mathrm{N}$ - and $\mathrm{C}$-terminal lobes of the kinase domain for the $\mathrm{S}$. scrofa sequence. Insertions in the kinase domains of GiPI3KI and GiPI3K2 are shown in boxes (not to scale). Solid underlined residues indicate activation loop residues in the $S$. scrofa sequence essential for substrate specificity. Dashed underlined residues indicate catalytic loop residues in the $S$. scrofa sequence. Key: $\diamond$, ATP binding residues; $\boldsymbol{\Delta}$, PI-binding residues; $\bigcirc$, metal binding residues; $\square$, possible substrate-activating base/nucleophile to attack $\gamma$-phosphate of ATP.

no evidence for the splicing of the insertion-encoding 


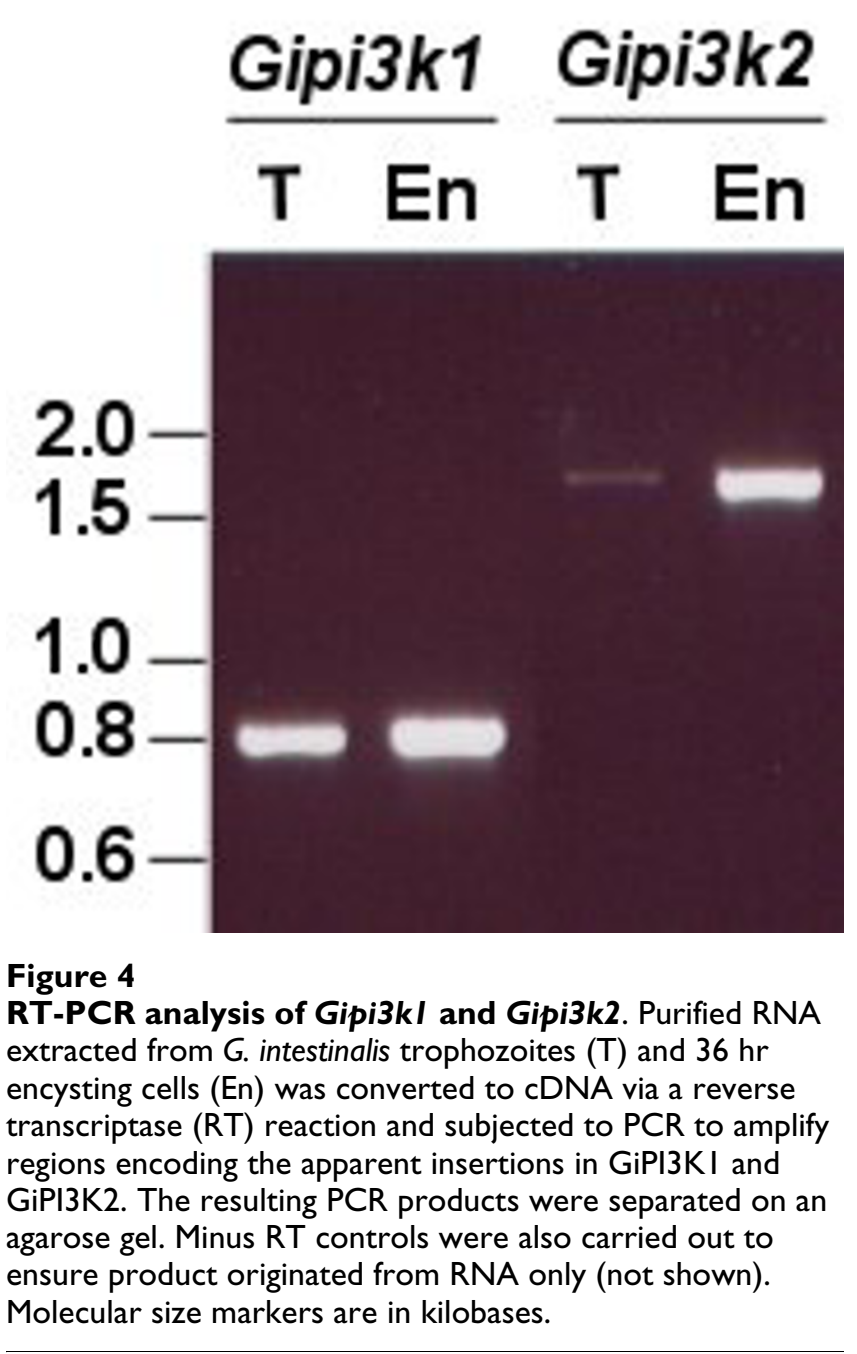

sequences of Gipi3k1 or Gipi3k2 RNA during parasite differentiation (Figure 4).

\section{Phylogenetic analysis of GiPI3KI and GiPI3K2}

Phylogenetic analyses of the two putative giardial PI3Ks clearly show their distinctive nature (Figure 5). GiPI3K1 clusters with the well characterised D. discoideum PIK1. Together, they are part of a clade exclusively containing PI3K1 homologs further strengthening our prediction that GiPI3K1 is a Class I isoform. The GiPI3K2 sequence belongs to a well-supported clade containing only Class III homologs, in agreement with our prediction based on domain composition (see Figure 1 and previous section). Although crown taxa fall in well-supported clades for the Class III homologs, support for the placement of the other sequences is lacking. The G. intestinalis Class III homolog is either basal to all other Class III homologs (Fig. 5A) or within the alveolates (Fig. 5B). The lengths of the branches leading to the G. intestinalis homologs are the longest in the analyses indicative of an accelerated evolution of these sequences. Phylogenetic reconstruction artefacts such as Long Branch Attraction [21] are therefore expected to affect these analyses. This would most likely only affect the placement within the isoform clade but not across isoforms. Therefore, our analyses cannot say much about the evolution of the G. intestinalis PI3Ks but can be used to identify which isoform each sequence belongs to.

\section{Characterisation of Gipi3k I and Gipi3k2 gene products}

RT-PCR analysis (Figure 4) demonstrated that Gipi3k1 and Gipi3k2 are both expressed in trophozoites and $36 \mathrm{hr}$ encysting cells, suggesting they play an important role during growth and possibly during encystation. The predicted open reading frames of Gipi3k1 and Gipi3k2 encode longer amino acid sequences as compared with other well-studied Class I and III PI3Ks (Figure 1). To test the coding capacity of these genes, we probed northern blots of RNA derived from trophozoites and $36 \mathrm{hr}$ encysting cells with sequences specific to the highly conserved kinase domain-encoding region of Gipi3k1 and Gipi3k2. We were unable to detect Gipi3k2 mRNA despite using control probes against well-characterised giardial transcripts to confirm RNA integrity (data not shown). This may indicate that the Gipi3k2 message is relatively unabundant, detectable only by sensitive techniques such as RT-PCR. The apparent Gipi3k1 mRNA transcript detected by northern blotting (Figure 6) was smaller than expected based on the predicted size of the Gipi3k1 open reading frame: $3.5-4 \mathrm{~kb}$ as opposed to $\sim 6.5 \mathrm{~kb}$. Subsequent 5'RACE experiments (Figure 7) indicated that three transcriptional initiation sites of the Gipi3k1 gene may exist, capable of producing transcripts of $\sim 6.5 \mathrm{~kb}, \sim 5 \mathrm{~kb}$, and $\sim 3.8 \mathrm{~kb}$ in length, assuming they shared a common $3^{\prime}$ polyadenylation site. A typical giardial polyadenylation site conforming to the consensus AGTPurAAPyr [22] for the Gipi3k1 gene was found (AGTATAAT), however putative polyadenylation sites for Gipi3k2 could not be identified. The shortest Gipi3k1 5'RACE product could represent the Gipi3k1 transcript detected by northern blotting. This suggests that the major transcript of the Gipi3k1 gene would encode a product more akin to the molecular weights observed for other well-studied PI3Ks [11], although it would lack the potential RBD. This may imply the existence of protein products which not only differ in size but also in the way in which they are regulated. The RBD of a Class I PI3K has been shown to stimulate the PI3K's kinase activity in addition to stabilising its association with the plasma membrane [23]. Thus Gipi3k1 protein products with an RBD may display differences in activity and/or localisation compared to those without an RBD.

Possible giardial promoter-like elements found upstream from each of the three alternative Gipi3k1 transcripts are 
A

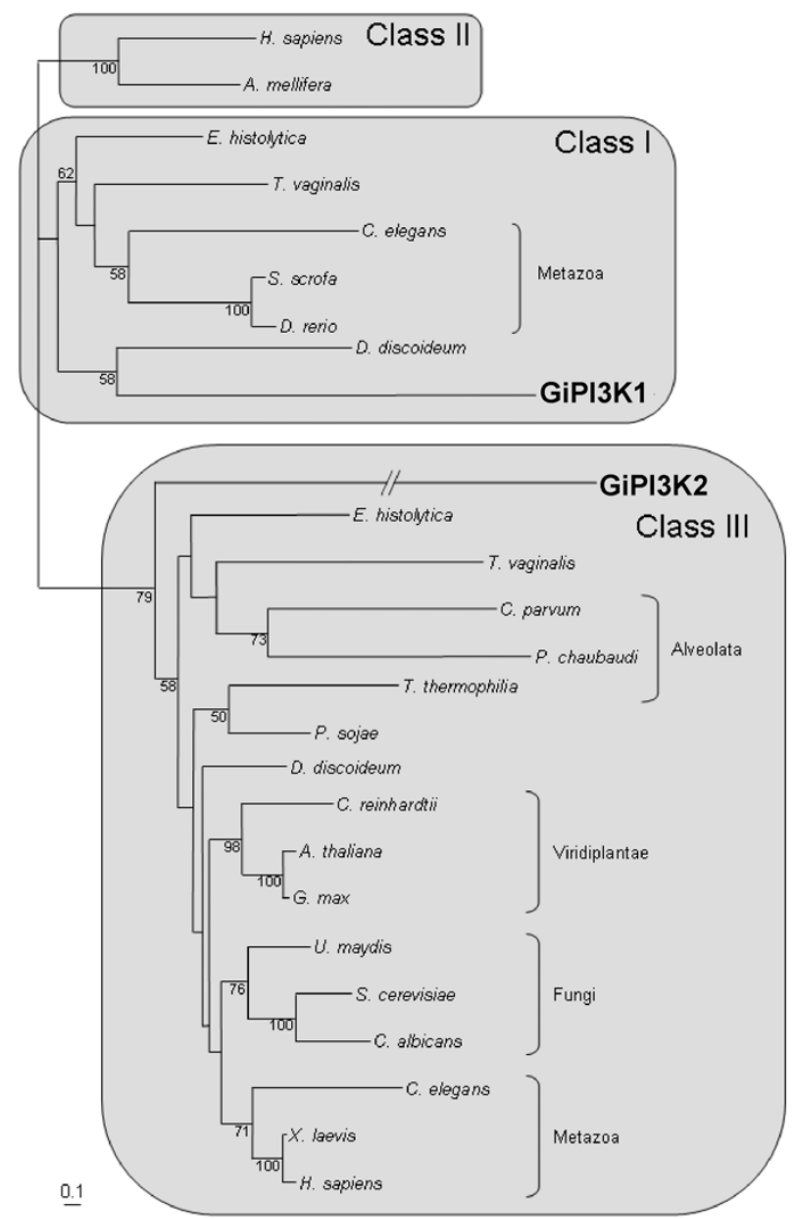

B

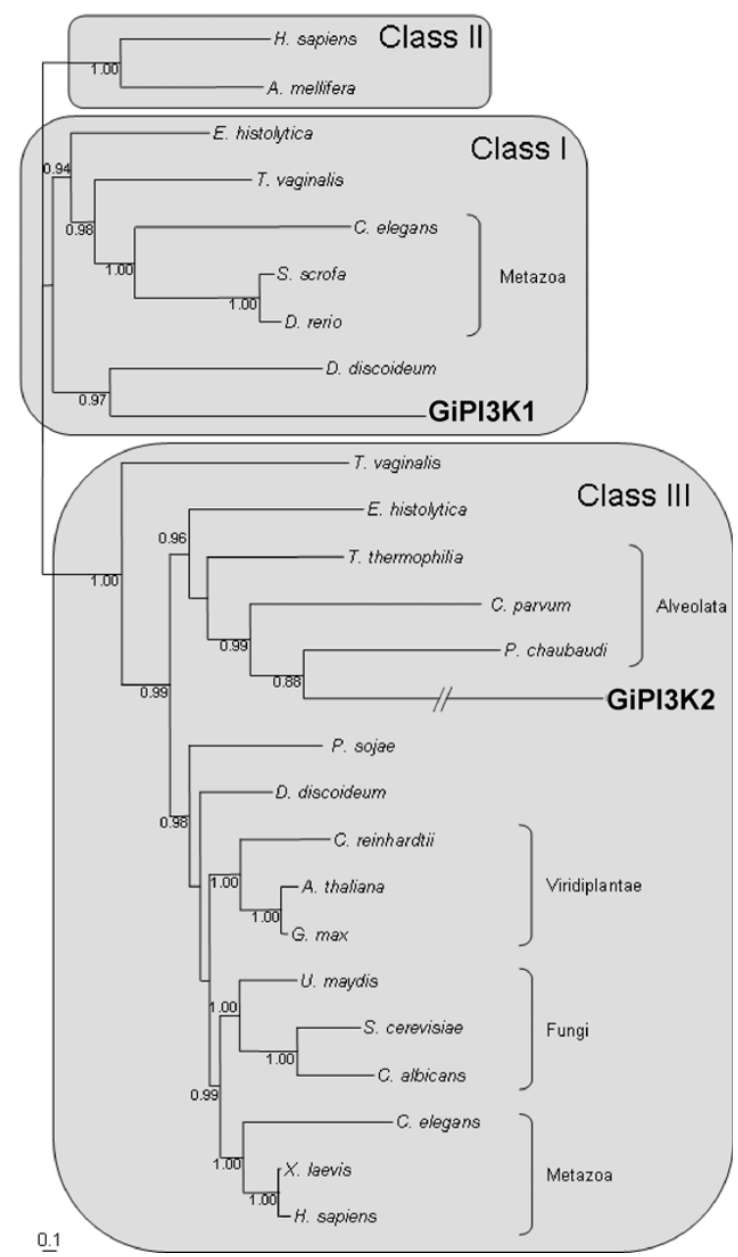

Figure 5

Phylogenetic analysis of G. intestinalis PI3K homologs. Phylogenetic analyses of the different classes of PI3Ks including GiPI3KI and GiPI3K2 (shown in the Class I and Class III clusters respectively). Shown are unrooted trees of representative sequences of Class I, Class II and Class III PI3Ks. A, Maximum likelihood analysis using PHYML [40]. Bootstrap values based on 100 replicates indicated at the nodes, only values above $50 \%$ are shown. B, Bayesian analysis of the same dataset using MrBayes v3.I [39], posterior probabilities, calculated by MrBayes are indicated at the nodes, only values above 0.75 are shown. The branches leading to GiPI3K2 have been shortened by one third.

also indicated in Figure 7. Promoter elements in G. intestinalis tend to have a relatively loose consensus, however it is generally thought that an AT-rich initiator of 8-10 bp exists near the transcription/translation start site in addition to a longer AT-rich element further upstream from the transcriptional start site which may contain CAAT boxes [24]. It is interesting that the apparent promoters of all three Gipi3k1 transcripts have some or all of these features. In addition, the shortest transcript is the only one to have upstream CAAT boxes. Possibly the 'loose' molecular machinery controlling giardial transcription [25] could be responsible for the presence of three Gipi3k1 transcripts, whereby transcription is initiated at several AT-rich regions. The use of specific antibodies against GiPI3K1 will be required to clarify whether the transcripts encode multiple proteins with distinct functions.

\section{Effect of PI3K inhibition on trophozoite proliferation}

To determine the functional role of putative PI3Ks in G. intestinalis growth, we applied a commonly used PI3K inhibitor, LY294002, on G. intestinalis trophozoites. We tested a range of concentrations around those shown to selectively inhibit mammalian PI3Ks [26]. Figure 8a shows a dose-response of exponentially growing cells to the inhibitor, with concentrations of LY294002 as low as $25 \mu \mathrm{M}$ causing a significant inhibitory effect on cell number as compared with the untreated control. Approximately $50 \%$ inhibition of cell proliferation occurred at 


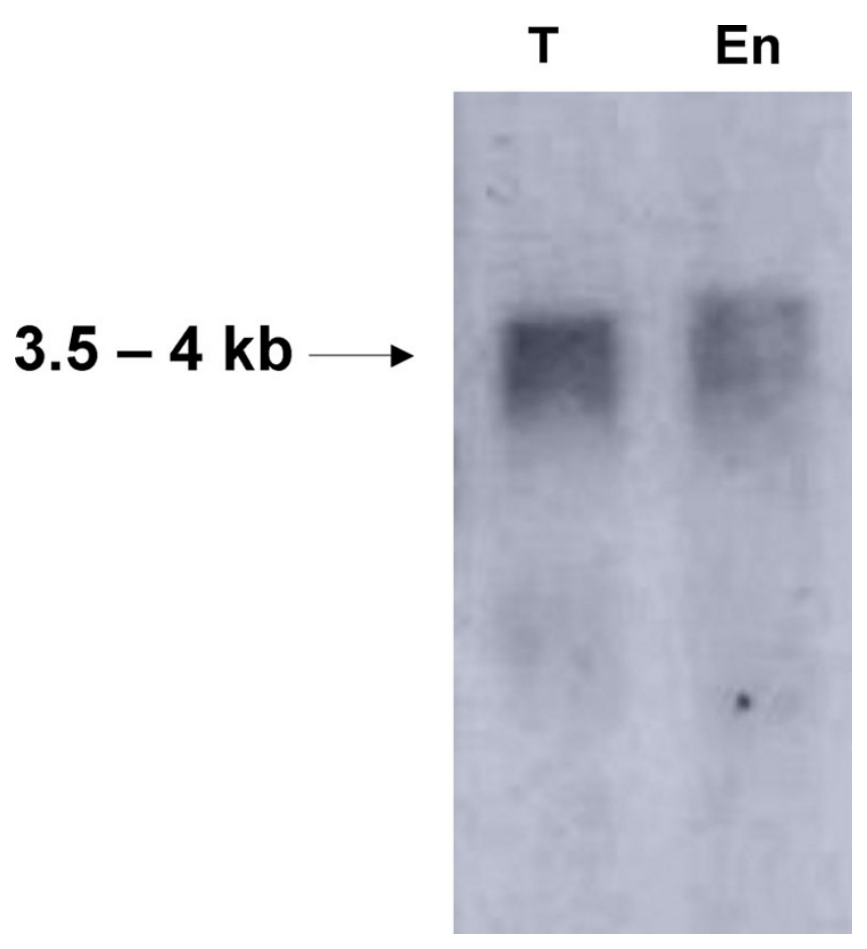

\section{Figure 6}

Northern blot of Gipi3kI RNA in trophozoites and encysting cells. Northern blotting of trophozoite $(T)$ and encysting cell (En) total RNA, as described in the Methods section, was carried out using a probe specific to Gipi3k I RNA sequence to identify transcripts of approximately 3.5 4 kilobases.

concentrations between 25 and $75 \mu \mathrm{M}$. This effect is likely to be PI3K-mediated, since LY294002 concentrations within the $50-100 \mu \mathrm{M}$ range have been employed for selective PI3K inhibition in mammalian cells. To understand the time course over which LY294002 exhibited its effects, we counted $50 \mu \mathrm{M}$ LY294002 - treated cells at regular intervals over a 48 hour period. Figure $8 \mathrm{~b}$ demonstrates that LY294002 begins to significantly effect cell number 8 hours into treatment. For the duration of the time-course, cell number remains approximately constant, whilst the untreated control continues to grow exponentially. This suggests that LY294002 may affect cell proliferation by inducing cell cycle arrest. In addition, trophozoites treated with LY294002 do not undergo any dramatic changes in their morphology or motility, thus further demonstrating the selective effect of LY294002 on Giardia's cell cycle.

To ensure that the effects of LY294002 were due to inhibition of one or more of the putative PI3Ks and not another target, such as the Casein Kinase II (CKII) protein which can also be inhibited by LY294002 [26], we tested the effect of the CKII-inhibitor, DRB (5,6-Dichloro-1- $\beta$-D- ribofuranosylbenzimidazole), on trophozoite proliferation. Putative giardial CKII may exist under the accession numbers XP_766966 (for the alpha subunit) and EAA39338 (for the beta subunit), although there is no experimental evidence for this inference. The use of DRB at concentrations known to be inhibitory in mammalian cell types [27] did not cause the same effect on cell proliferation as LY294002 (Figure 8b). This, coupled with the fact that relatively low concentrations of LY294002 cause a significant decrease in cell number, strongly supports a specific effect on putative PI3Ks. Furthermore, this data suggests that trophozoite proliferation is dependent on the functionality of PI3K signalling.

\section{Discussion}

Our study has identified and characterised two putative and distinctive giardial PI3K-encoding genes and gene products. GiPI3K1 is predicted to be a Class I PI3K and GiPI3K2 a Class III PI3K; both are predicted to be functional as PI3Ks and both are expressed during normal growth and possibly during encystation. In addition, we have demonstrated that inhibition of putative giardial PI3Ks by the PI3K inhibitor LY294002 causes a specific and significant inhibition of trophozoite proliferation.

Interestingly, the PI3K inhibitor wortmannin did not effect trophozoite growth as LY294002 did. This was despite our attempts to account for wortmannin instability $[28,29]$ by both testing the activity of our stock solutions on mammalian cell cultures to detect reduced phosphorylated PKB levels and by making repeated additions of the agent to trophozoite cultures (data not shown). Wortmannin-insensitive PI3Ks have been described in yeast, where yeast Vps34 is known to be $1200 \times$ less susceptible to wortmannin inhibition than its human homologue [30]. Differences in sensitivity can be explained by differences in key ATP/wortmannin-binding regions of the respective Class III isoforms [30]. Analyses of the giardial PI3Ks demonstrate that they too have residues that differ at the same positions described for yeast Vps34. For example, human Vps34 and yeast Vps34 differ at positions equivalent to positions Ile-831 and Gly-868 in the S. scrofa sequence in Figure 3. At these positions, the isoleucine is replaced by a leucine and the glycine replaced with a serine in both GiPI3K1 and GiPI3K2. These residue differences might explain Giardia's apparent insensitivity to wortmannin. An alternative explanation is that the insertions located near to the active sites of GiPI3K1 and GiPI3K2 somehow interfere with wortmannin binding, but not LY294002 binding, since the inhibitory mechanisms of both inhibitors are quite distinct. Wortmannin is an irreversible inhibitor that causes conformational changes to the PI3K active site, whereas LY294002 is a reversible inhibitor that binds less deeply into the active site than wortmannin [30]. Further analysis 


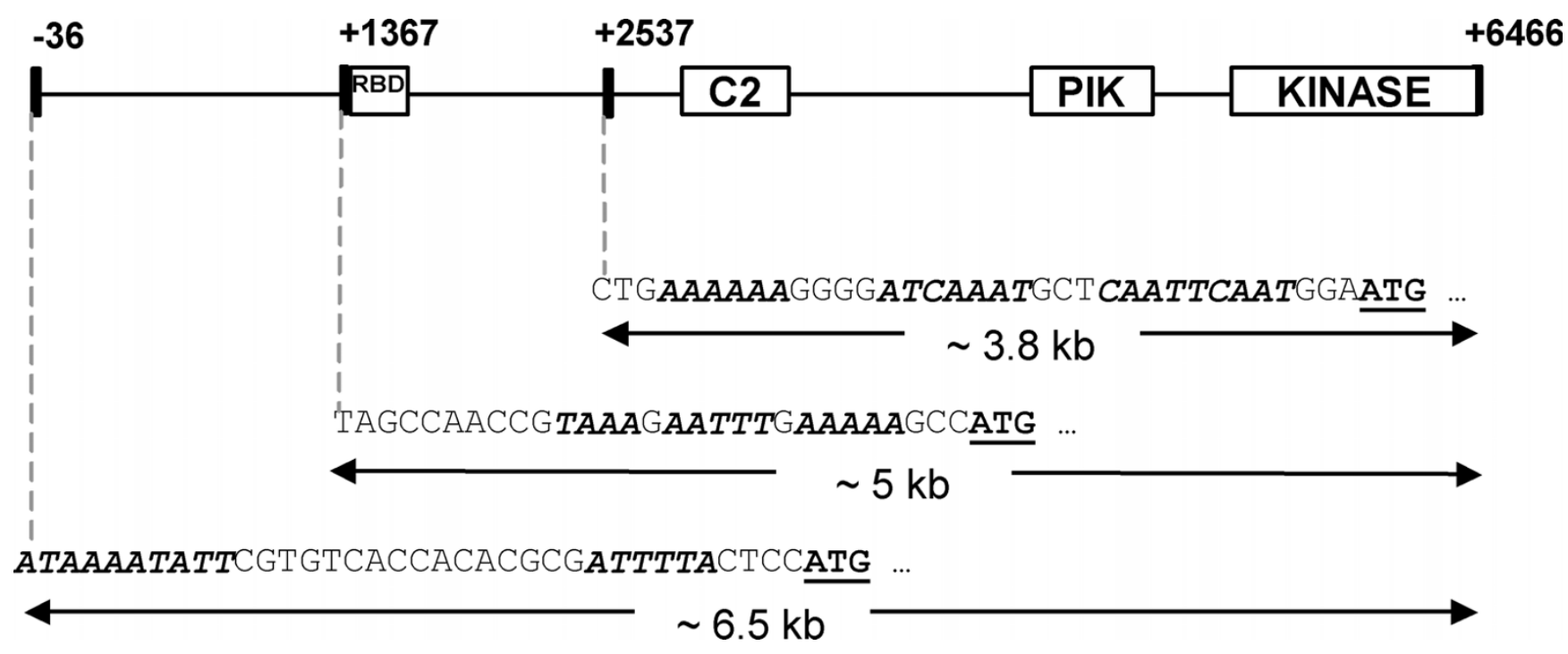

Figure 7

Analysis of transcriptional initiation sites of the Gipi3kl gene. 5' RACE analysis, performed as described in the Methods section, suggests the presence of multiple transcription initiation sites for the Gipi3k I gene. Sequence motifs common in $G$. intestinalis promoters are in italics and bold. Putative start codons are in bold and underlined. Predicted sizes of three Gipi3k $I$ mRNA transcripts are shown.

will be required to test the wortmannin sensitivity of purified giardial PI3Ks.

The giardial PI3Ks contain long insertions which effectively appear to separate the two lobes of their putative kinase domains. These insertions are unique to the giardial sequences and are likely to form part of the protein sequence, as they are not intron-derived. The reason for their existence is unknown, but could simply represent a peculiar feature of the evolutionarily distant/parasiticallydivergent $G$. intestinalis. The presence of the giardial PI3K insertions further adds to the observation of relatively large insertions of unknown function found in protein kinases from other organisms such as Plasmodium, Leishmania and trypanosomes [31] in addition to other giardial sequences [32]. Insertions in giardial sequences have been suggested to serve as useful drug targets [32]. This is particularly relevant in the case of the giardial PI3Ks identified here since PI3K activity in G. intestinalis is important for parasite proliferation. Hence drugs that could selectively target giardial PI3Ks on the basis of their insertion sequences would be highly desirable in terms of being parasite-specific and effective. Current progress in designing PI3K-directed pharmacological agents [33] suggests that this could be attainable in the future.

The identity of Class I and Class III isoforms suggests that G. intestinalis is capable of PI-based signal transduction for a range of processes covering typical Class I PI3K sites (the plasma membrane and cytosol for receiving and process- ing extracellular signals) and Class III PI3K sites (intracellular membranes for regulation of vesicle trafficking). To understand the potential role of PI3K signalling in G. intestinalis in a broader context, we used sequence-similarity tools to identify additional giardial signalling homologues. Figure 9 shows giardial proteins which could metabolise the putative products of GiPI3K1 and GiPI3K2 in addition to other PI intermediates, starting with phosphatidylinositol which has already been found in G. intestinalis [8]. The PI-metabolising phosphatases identified include: a PI 5-phosphatase and a PTEN-like PI 3-phosphatase which may both act to dephosphorylate the likely products of GiPI3K1 in addition to an MTM-like PI 3phosphatase that could dephosphorylate the likely product of GiPI3K1 and GiPI3K2. The PI-metabolising kinases identified include: a PIPkin, or phosphatidylinositolphosphate kinase of as yet unknown type which could phosphorylate several PI-intermediates, such as PtdIns(3)P, PtdIns(3,4) $\mathrm{P}_{2}$ and PtdIns(4)P in addition to a putative PI-4 kinase to phosphorylate PtdIns for the purpose of eventual PtdIns $(3,4,5) \mathrm{P}_{3}$ generation. Thus sequence analysis does support mechanisms for metabolising the products of the giardial PI3Ks.

To predict how these products, PtdIns(3)P, PtdIns $(3,4) \mathrm{P}_{2}$ and PtdIns $(3,4,5) \mathrm{P}_{3}$, may act as effective second messengers for downstream effectors, we searched the G. intestinalis genome [16] for proteins which have the relevant PIbinding domains such as phox-homology (PX), FYVE (Fab1, YOTB/ZK632.12, Vac1, and EEA1) and pleckstrin- 
a.

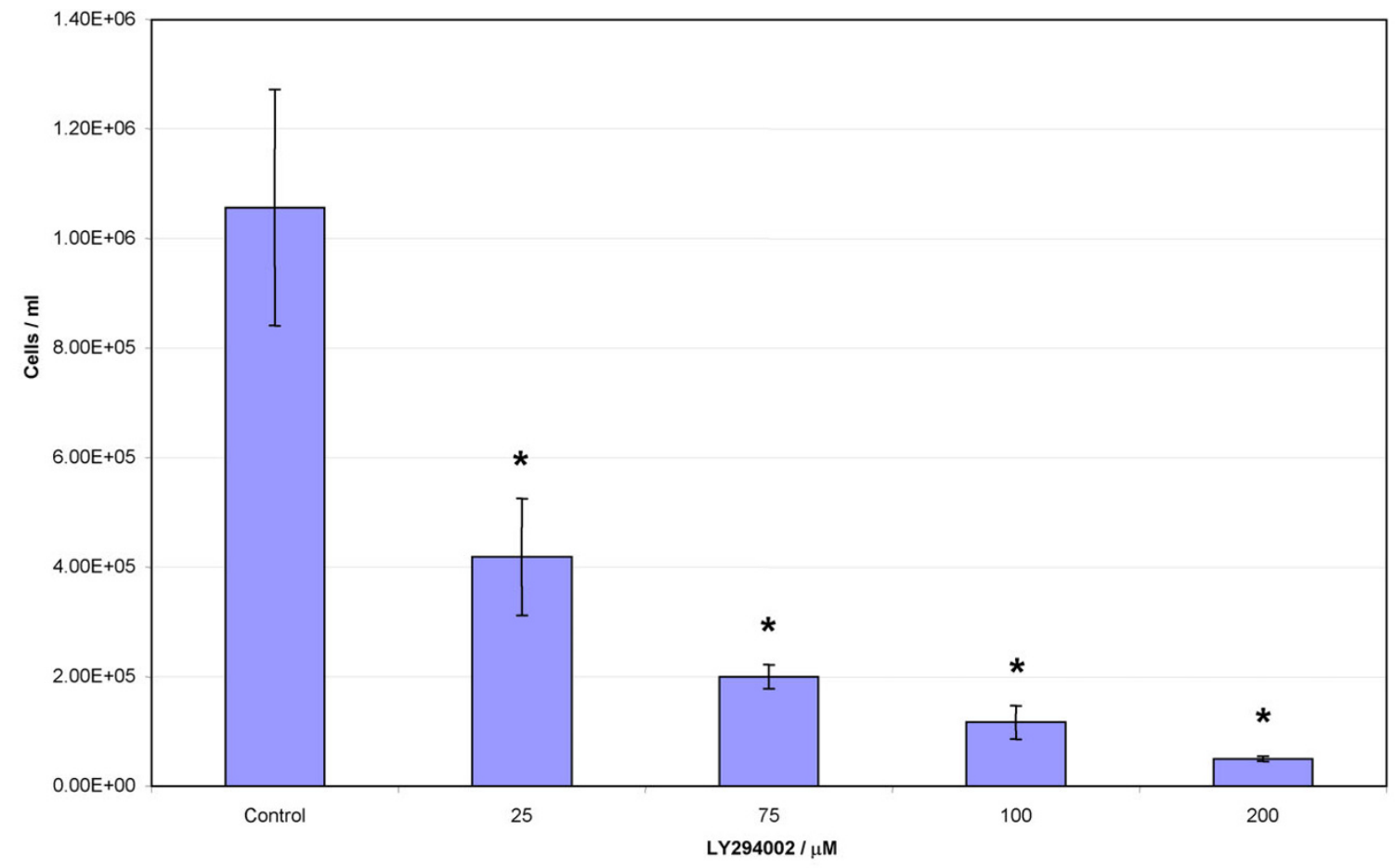

b.

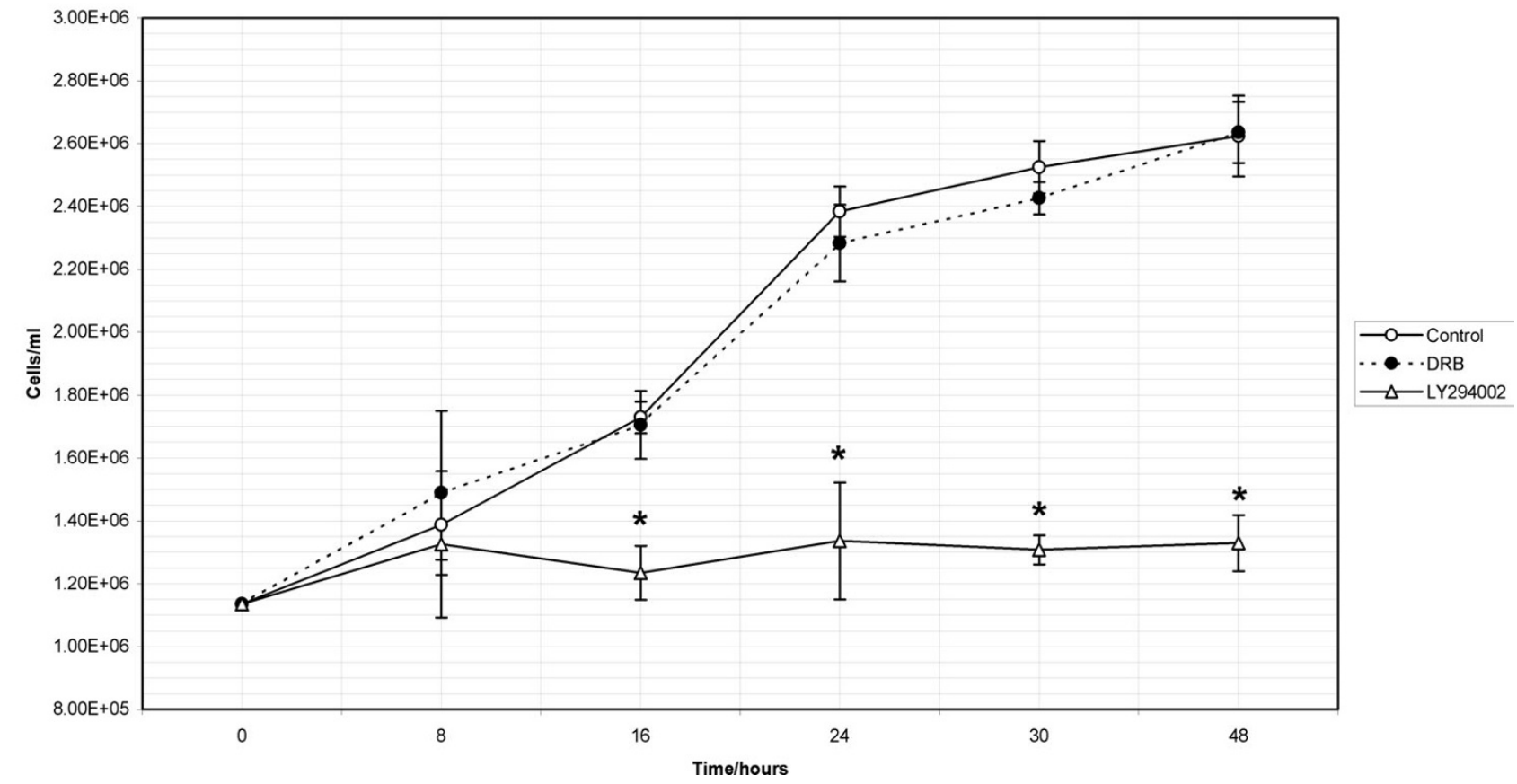

Figure 8

Inhibition of trophozoite proliferation by a PI3K inhibitor. a) Trophozoites treated with increasing concentrations of LY294002 were counted after 48 hours of treatment. b) To test the specificity of inhibition, the effect of LY294002 (50 $\mu$ M) and DRB ( $100 \mu \mathrm{M}$ final concentration) on exponentially growing trophozoites was monitored by cell counting over a 48 hour period. Error bars indicate the standard deviation of 3 counts. Asterisks denote significance at $p<0.00$ I for normalised data. 


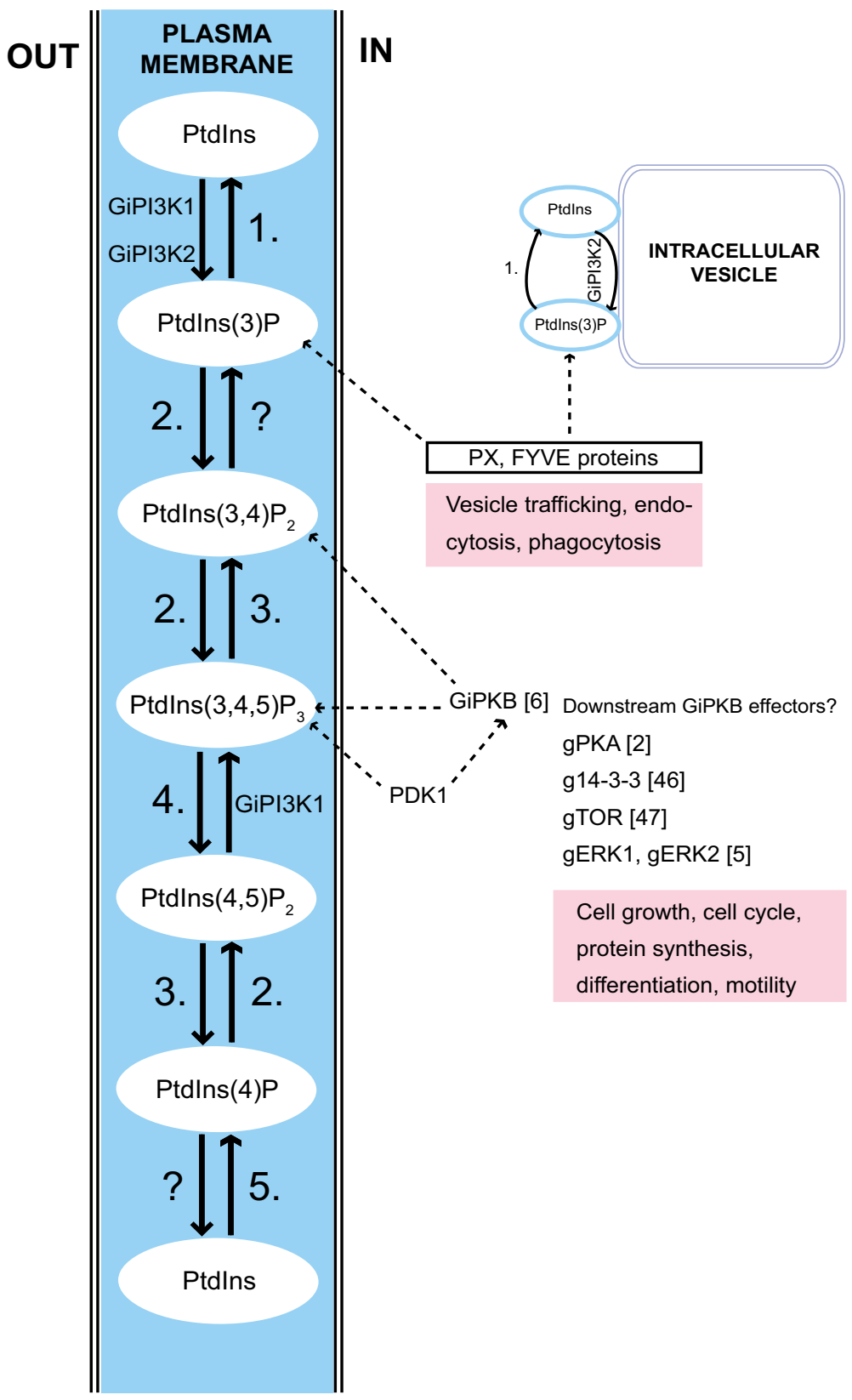

Figure 9

Proposed model for PI metabolism and PI3K signalling in G. intestinalis. A model of PI3K signalling in G. intestinalis based on known mechanisms in mammalian cells and yeast. GiPI3KI and GiPI3K2 are suggested to localise at the plasma membrane and GiPI3K2 at some undefined intracellular compartment. Proteins that may participate in G. intestinalis PI metabolism have been identified and are shown (as indicated by numbering): (I) MTM (Myotubularin, GenPept: EAA37232 or EAA38552), (2) PIPkin (Phosphatidylinositol phosphate kinase, GenPept: EAA42556), (3) PI 5-phosphatase (GenPept: EAA40I47), (4) PTEN (Phosphatase and tensin homolog, GenPept: EAA42276), (5) PI4K (Phosphoinositide 4-kinase, GenPept: EAA38I2I). The question mark indicates a possible 4-phosphatase of unknown identity. Ptdlns(3)P possibly recruits PX and FYVE -domain-containing proteins, of which there are several encoded in the $G$. intestinalis genome (PX-domain proteins: EAA39579, EAA42255, EAA428I I, EAA42799 are possible sorting nexins; FYVE-domain protein: EAA42608, a possible endofin). A putative giardial PDKI (3-phosphoinositide-dependent protein kinase, GenPept: EAA38977) may be recruited to Ptdlns $(3,4,5) P_{3}$. Other previously characterised giardial signalling components which may be invoked could include: GiPKB [6], gPKA [2], gl4-3-3 [46], gTOR [47] and gERKI and gERK2 [5]. Not shown in this figure is the possible generation of Ptdlns $(3,4) P_{2}$ from Ptdlns $(4) P$ by GiPI3KI. 
homology (PH). PH-domain containing proteins such as protein kinase $\mathrm{B}$ (PKB) use their PH domain to facilitate recruitment to the plasma membrane where PtdIns $(3,4,5) \mathrm{P}_{3}$ is located. Although Kim et al. (2005) failed to identify a PH domain in the giardial PKB, GiPKB, with significant similarity to the $\mathrm{PH}$ domain of other PKBs, the percentage identity and similarity scores following our own sequence alignment of GiPKB's putative $\mathrm{PH}$ domain with that of human PKB were 12 and $36 \%$ respectively (data not shown). Thus it may be that GiPKB is capable of binding PtdIns $(3,4,5) \mathrm{P}_{3}$ via a putative divergent PH domain. Another protein that binds PtdIns $(3,4,5) \mathrm{P}_{3}$ via its $\mathrm{PH}$ domain is 3-phosphoinositidedependent kinase-1 (PDK1) which phosphorylates PKB. We were able to identify a putative giardial PDK1 homologue which did not have an easily identifiable $\mathrm{PH}$ domain when employing domain-prediction tools. However upon pairwise-alignment of its sequence with that of human PDK1, a PH domain-like sequence was identified with $23 \%$ identity and $51 \%$ similarity (data not shown).

Figure 9 proposes that from GiPKB, several other regulatory pathways involving protein components previously characterised in G. intestinalis could be initiated. We were able to identify PX- and FYVE-domain containing proteins encoded by the G. intestinalis genome [16]. In mammalian cells these domains facilitate binding and recruitment to PtdIns(3)P. The giardial PX-domain proteins tend to be similar to sorting nexins, and the FYVE-domain protein is similar to endofin. Both of these proteins are implicated in regulating membrane traffic. Thus via these giardial homologs it may be possible to connect the product of GiPI3K2 with vesicle trafficking mechanisms. Overall, the presence of putatively functional PI3Ks, PI-metabolising enzymes and downstream effectors further substantiates the existence of bona-fide PI3K signalling pathways in G. intestinalis, as supported by our functional data employing a widely-used and characterised PI3K inhibitor.

\section{Conclusion}

We conclude that the human intestinal parasite G. intestinalis encodes and expresses two putative PI3Ks, GiPI3K1 and GiPI3K2, both of which are likely to be functional based on sequence analysis. Functionality of PI3Kdependent processes has been demonstrated by observing the specific inhibitory effect of a PI3K-inhibitor on trophozoite proliferation. Phylogenetic analysis supports the assignment of GiPI3K1 and GiPI3K2 to the Class I and Class III PI3K subdomains respectively, and in-depth sequence analysis further substantiates their proposed substrate specificities. Genome sequence analysis can link the products of GiPI3K1 and GiPI3K2 with putative giardial PI-metabolising phosphatases and kinases, in addition to downstream effectors with PI-binding domains.
This provides additional evidence for bona-fide PI3K signalling pathways in G. intestinalis.

A feature unique to both giardial PI3Ks is the presence of a large insertion in a highly conserved region of their kinase domains. Both insertions are not subject to splicing and are not predicted to interfere with substrate binding. Thus, considering the important role of PI3Ks for parasite proliferation, it is possible that the insertion sequences could be exploited for drug-targeting purposes.

\section{Methods}

\section{Sequence similarity searches and alignments}

The translation BLAST [34] tool at the National Center for Biotechnology Information (NCBI) was used with default settings to search for putative PI3Ks in the Giardia intestinalis genome [16] using the kinase domain sequences of the following protein sequences as queries: Homo sapiens p110 $\alpha$ [GenPept: NP_006209], H. sapiens PI3K-C2 $\alpha$ [GenPept: NP_002636] and Saccharomyces cerevisiae Vps34 [GenPept: NP_013341]. The searches returned several matches to putative open reading frames (ORFs). Gene sequences in a non-redundant database at NCBI were searched for similar sequences to the putative ORFs via NCBI's translation-BLAST and nucleotide-BLAST tools (used with default settings) in order to identify two putative PI3K-encoding ORFs (subsequently called Gipi3k1 and Gipi3k2). SMART [35], and SCOP [36] were used with default settings to predict PI3K-characteristic domains in GiPI3K1 and GiPI3K2 and other PI3Ks. Several sequences were multiple-aligned using ClustalW [37] with default settings. Secondary structure predictions were carried out using the PSIPRED server [38] used with default settings.

\section{Phylogenetic analysis}

The conceptually translated G. intestinalis PI3K sequences, GiPI3K1 and GiPI3K2, were aligned to reference sequences from Genbank using ClustalW [37]. In order to obtain a taxonomically sound sample, homologues sequences were sought amongst the Metazoa, Viridiplantae, Fungi, Stramenopiles, Alveolates, and species generally considered to be 'deep-branching'. The alignments were manually refined and only unambiguously aligned regions without gaps were used for phylogenetic analysis, leaving a dataset of 26 taxa with 473 amino acid positions. Likelihood searches were performed in a Bayesian framework using mixed amino acid models accommodating site rate variation (fraction of invariable sites plus eight variable gamma rates) using the program MrBayes [39]. All analyses started with randomly generated trees and ran for 100,000 generations, with sampling at intervals of 100 generations that produced 1,000 trees. The log-likelihood values of the 1,000 trees were plotted against the generation time (not shown). All the trees produced prior to reaching stationarity were discarded, mak- 
ing sure that burn-in samples were not retained. Although the likelihood model stabilized very rapidly (data not shown), only the last 900 trees were used to estimate separate $50 \%$ majority rule consensus trees for these. The frequency of any particular clade, among the individual trees contributing to the consensus tree, represents the posterior probability of that clade [39]. In addition, maximum likelihood (ML) analyses were obtained using PHYML [40]. The protein data set was re-sampled 100 times and analysed with alpha and invariant sites parameters optimised on the initial BIONJ tree produced by PHYML. Analyses were done with a mixed eight-category discrete gamma plus invariable sites model of rate heterogeneity. The JTT substitution model was used in the protein analyses. Majority rule consensus trees were obtained from the resulting 100 trees.

\section{G. intestinalis cell culture}

G. intestinalis strain WB (ATCC 30957, clone C6) was routinely subcultured every 3 to 4 days at $37^{\circ} \mathrm{C}$ in axenic modified YI-S medium [41]. Encystation was carried out according to published procedures [42].

\section{PI3K inhibition}

Routinely subcultured $G$. intestinalis trophozoites were grown for 48 hours prior to treatment with the PI3K inhibitor LY294002 (2-(4-Morpholinyl)-8-phenyl-4H-1benzopyran-4-one hydrochloride) and CKII inhibitor DRB (5,6-Dichloro-1- $\beta$-D-ribofuranosylbenzimidazole). Cells were treated with LY294002 to achieve final concentrations of $25,50,75,100$ and $200 \mu \mathrm{M}$ from a $100 \times$ stock solution in DMSO. Cells were treated with DRB to achieve a final concentration of $100 \mu \mathrm{M}$ from a $100 \times$ stock solution. Control cells were treated with equal volumes of DMSO. Experiments were carried out in triplicate. Cells were fixed in $4 \%$ paraformaldehyde (Sigma) and counted with a haemocytometer. Count data was tested for normal distribution and significance using the Student t-test within the SigmaPlot package (Systat Software Inc.).

\section{Nucleic acid methods \\ DNA and RNA isolation}

Genomic DNA was extracted from harvested trophozoites according to the TriPure Isolation method (Roche). Total RNA was extracted from both harvested trophozoites and encysting cells using the RNAeasy Mini Kit for animal cells (Qiagen). RNA was purified from contaminating DNA using DNase I (Invitrogen) and the RNAeasy Mini Protocol for RNA Cleanup (Qiagen) was used to remove components of the DNase I digestion reaction from RNA.

\section{Generation of cDNA}

Total RNA was reverse-transcribed into cDNA in a $20 \mu \mathrm{l}$ reaction mix containing $6 \mu \mathrm{g}$ total RNA, $5 \mu \mathrm{M}$ random hexamer primers, $2.5 \mathrm{mM}$ DTT, $250 \mu \mathrm{M}$ dNTPs, $40 \mathrm{U}$
RNaseOUT recombinant ribonuclease inhibitor (all from GibcoBRL) and 25 U MLV RT (GeneSys Ltd). RNA was denatured by heating at $70^{\circ} \mathrm{C} 10 \mathrm{~min}$, followed by cooling on ice to anneal random hexamer primers. MLV RT was added, and incubated at $37^{\circ} \mathrm{C}$ for 1 hour. Negative controls replaced RT with RNAse-free water.

\section{Polymerase Chain Reaction (PCR)}

PCR was performed on G. intestinalis cDNA, genomic DNA and plasmid DNA using the following gene specific primers: Gipi3k1 ins_5465S: (5' GCC ATT CGA GGG TTT GAT AG 3'); Gipi3k1 ins_6216AS: (5' GAT TGC TAG TAG GGC ATC TG 3'); Gipi3k2 4871AS: (5' TGT GGA AGG CGT CGA TGA AC 3'); Gipi3k2 ins_3424S: (5' ACG ATG TGC GAC TAG ACA TG 3'); Gipi3k1(1)NotI S: (5' GAG AGC GGC CGC ACC ATG GGA ATG CAG GTT TCC ATT ATA AG 3'); Gipi3k1(1)BamHI AS: (5' CTC TGT AGG TGG CTT ATA TG 3'); Gipi3k1(2)BamHI S: (5'CTA TGC GCA TAA GCT TTG AG 3'); Gipi3k1(2) BamHI AS (5' GGA AGC GCC TCC TCC AAC TCT AAG GAT CCG AGA 3'). Primer sequences designed to demonstrate the presence of the intron in some G. intestinalis ferredoxin-encoding mRNAs were taken from Nixon et al. (2002) [43] and included as a positive control to confirm the presence of spliced and unspliced mRNA. Gipi3k1 and Gipi3k2 PCR products (generated from primers Gipi3k1 5945S/6374AS and Gipi3k2 4441S/4871AS respectively) were cloned into pGEM-T Easy (Promega) and sequenced using the dideoxy-nucleotide termination method at the DNA Analysis Facility, Dundee University. Sequence output was analysed and verified using BioEdit [44].

\section{Rapid Amplification of CDNA Ends (RACE)}

The 5' RACE system from Invitrogen was used to determine the start of Gipi3k1 transcription. Oligonucleotides GSP1_393 (5' GCT GAC AAG AGC CTC TGC TA 3') or GSP1_2947 (5' GGC TTT AGA AGA AAC AAG GA 3') primed the first strand CDNA synthesis, and oligonucleotides GSP2_313 (5' GCC AGC TTT CTA GCA GGT CA 3') or GSP2_2914 (5' GTC CAC TTG AGA AGT ATC CT 3') served as nested primers. PCR products were cloned and sequenced as above.

\section{Northern blotting}

Northern blotting was carried out using a nonradioactive method (Roche). DNA probes incorporating digoxigenin (DIG) were generated against the kinase-domain encoding region of Gipi3k1/2 via PCR amplification of pGEM-T Easy plasmids (Promega) carrying sequences of Gipi3k1/2 genes. $5 \mu \mathrm{g} \mathrm{G}$. intestinalis total RNA was prepared as described above and separated on a $1.2 \%$ agarose $/ 0.5 \%$ formaldehyde/1\% $1 \times$ MOPS (3-M-Morpholino propanesulphonic acid) gel and transferred to positively charged nylon membrane (BrightStar-Plus, Ambion) by capillary transfer. RNA was fixed to the membrane by bak- 
ing at $80^{\circ} \mathrm{C}$ for $2 \mathrm{hr}$. DIG-labelled DNA probes were hybridised to blots for 16 hours at $50^{\circ} \mathrm{C}$. Membranes were washed at high stringency using techniques described by Roche. Antibody solution (1:10,000 Anti-Digoxigenin$\mathrm{AP}$ ) (Roche, $75 \mathrm{mU} / \mathrm{ml}$ ) was added to the membrane for $30 \mathrm{~min}$ at room temperature. Chemiluminescent substrate CSPD (Roche) was added to the membrane and exposed to film.

\section{Cloning and sequencing of Gipi3k I}

The entire Gipi3k1 nucleotide sequence was cloned into the pcDNA 3.1/myc-His(-) B vector (Invitrogen) as follows: the 6,468 bp Gipi3k1 gene sequence was PCR-amplified using $P f u$ polymerase from $G$. intestinalis genomic DNA as two approximately-equal sized inserts using primer pairs as described above which would incorporate NotI and BamHI restriction sites, specifically 5' NotI - 3' BamHI for the first insert and 5' BamHI - 3'BamHI for the second. Inserts were ligated into NotI/BamHI (Promega) linearised vector using T4 DNA ligase (Promega), transformed and cloned in SURE Escherichia coli (Stratagene) cells and plasmid DNA extracted using a Qiagen miniprep kit. pcDNA3.1/myc-His(-) B - Gipi3k1 plasmid was sequenced across the entire length of the Gipi3k1 sequence as described above.

\section{Abbreviations}

PI3K: Phosphoinositide-3 kinase; PI4K: Phosphoinositide-4 kinase; PtdIns: Phosphatidylinositol; PI: Phosphoinositides; PtdIns(3)P: PtdIns-3-phosphate; PtdIns(4)P: PtdIns-4-phosphate; PtdIns(3,4) $\mathrm{P}_{2}$ : PtdIns3,4-bisphosphate; PtdIns $(4,5) \mathrm{P}_{2}$ : PtdIns-4,5-bisphosphate; PtdIns $(3,4,5) \mathrm{P}_{3}$ : PtdIns-3,4,5-trisphosphate; PIPkin: Phosphatidylinositol phosphate kinase; MTM: Myotubularin; PTEN: Phosphatase and tensin homolog; RT-PCR: Reverse Transcriptase Polymerase Chain Reaction; 5' RACE: 5' Rapid Amplification of cDNA Ends; RBD: Ras Binding Domain; GiPI3K1/2: Giardia intestinalis PI3K1/2; CKII: Casein Kinase II; DRB: 5,6-Dichloro-1- $\beta$ D-ribofuranosylbenzimidazole; LY294002: 2-(4-Morpholinyl)-8-phenyl-4H-1-benzopyran-4-one hydrochloride; PX: Phox homology; PDK1: 3-phosphoinositidedependent kinase-1; PKB: Protein kinase B; PH: Pleckstrin homology; FYVE: Fab1, YOTB/ZK632.12, Vac1, and EEA1.

\section{Authors' contributions}

SSEC carried out the sequence alignments, annotation and domain characterization, in addition to the cell culturing, molecular genetic and inhibition studies. SSEC also drafted the manuscript. MvdG carried out the phylogenetic analysis. SJT contributed toward the inhibition studies. All authors participated in the sequence alignments and domain characterization. MRC and JT conceived of the study, and participated in its design and coordination and helped to draft the manuscript. All authors read and approved the final manuscript.

\section{Acknowledgements}

The use of publicly available genome sequence data at the Sanger Institute, TIGR, MBL and JGI is acknowledged. We thank Neil Sommerville for maintaining cell cultures. This work was supported in part by a RHUL Thomas Holloway Studentship to S.C., a RHUL Research Strategy Fund grant to M.R.C. and by a Wellcome Trust grant (059845) to J.T.

\section{References}

I. Buret AG, Scott KG, Chin AC: Giardiasis: Pathophysiology and Pathogenesis. In Giardia, the Cosmopolitan Parasite Edited by: Olson BE, Olson AG and Wallis PM. Oxon, U.K., CABI Publishing; 2002:109 $-1126$.

2. Abel ES, Davids BJ, Robles LD, Loflin CE, Gillin FD, Chakrabarti R: Possible roles of protein kinase $A$ in cell motility and excystation of the early diverging eukaryote Giardia lamblia. J Biol Chem 200I, 276: 10320 - I10329.

3. Bernal RM, Tovar R, Santos JI, Munoz ML: Possible role of calmodulin in excystation of Giardia lamblia. Parasitol Res 1998, 84:687-693.

4. Reiner DS, Hetsko ML, Meszaros JG, Sun CH, Morrison HG, Brunton LL, Gillin FD: Calcium signaling in excystation of the early diverging eukaryote, Giardia lamblia. J Biol Chem 2003, 278:2533 -22540.

5. Ellis JG, Davila M, Chakrabarti R: Potential involvement of extracellular signal-regulated kinase $I$ and 2 in encystation of a primitive eukaryote, Giardia lamblia. Stage-specific activation and intracellular localization. I Biol Chem 2003, 278:1936 $-11945$.

6. Kim KT, Mok MTS, Edwards MR: Protein kinase B from Giardia intestinalis. Biochem Biophys Res Commun 2005, 334:333 -4I.

7. Lujan HD, Mowatt MR, Byrd LG, Nash TE: Cholesterol starvation induces differentiation of the intestinal parasite Giardia lamblia. Proc Natl Acad Sci 1996, 93:7628 -77633.

8. Ellis JE, Wyder MA, Jarroll EL, Kaneshiro ES: Changes in lipid composition during in vitro encystation and fatty acid desaturase activity of Giardia lamblia. Mol Biochem Parasitol 1996, 81:13 $-125$.

9. Subramanian $A B$, Navarro $S$, Carrasco RA, Marti M, Das S: Role of exogenous inositol and phosphatidylinositol in glycosylphosphatidylinositol anchor synthesis of GP49 by Giardia lamblia. Biochim Biophys Acta 2000, I 483:69 -680.

10. Vanhaesebroeck B, Leevers SJ, Ahmadi K, Timms J, Katso R, Driscoll PC, Woscholski R, Parker PJ, Waterfield MD: Synthesis and function of 3-phosphorylated inositol lipids. Annu Rev Biochem 200I, 70:535 -5602.

II. Foster FM, Traer C], Abraham SM, Fry MJ: The phosphoinositide (PI) 3-kinase family. J Cell Sci 2003, I I 6:3037-3040.

12. Cantrell DA: Phosphoinositide 3-kinase signalling pathways. J Cell Sci 200I, I | 4: |439- I I445.

13. Zhou K, Takegawa K, Emr SD, Firtel RA: A phosphatidylinositol (PI) kinase gene family in Dictyostelium discoideum: biological roles of putative mammalian pI 10 and yeast $\mathrm{Vps} 34 \mathrm{p} \mathrm{PI}$ 3-kinase homologs during growth and development. Mol Cell Biol 1995, 15:5645-55656.

14. Merlot S, Firtel RA: Leading the way: Directional sensing through phosphatidylinositol 3-kinase and other signaling pathways. J Cell Sci 2003, I 16:347। -33478.

15. Herman PK, Emr SD: Characterization of VPS34, a gene required for vacuolar protein sorting and vacuole segregation in Saccharomyces cerevisiae. Mol Cell Biol 1990, 10:6742 -66754 .

16. McArthur AG, Morrison HG, Nixon JE, Passamaneck NQ, Kim U, Hinkle G, Crocker MK, Holder ME, Farr R, Reich Cl, Olsen GE, Aley SB, Adam RD, Gillin FD, Sogin ML: The Giardia genome project database. FEMS Microbiol Lett 2000, 189:27I-273.

17. Field MC, Ali BRS, Field H: GTPases in Protozoan Parasites: Tools for Cell Biology and Chemotherapy. Parasitology Today 1999, 15:365-337I. 
18. Walker EH, Perisic O, Ried C, Stephens L, Williams RL: Structural insights into phosphoinositide 3-kinase catalysis and signalling. Nature 1999, 402:313 -3320.

19. Hetsko ML, McCaffery JM, Svard SG, Meng TC, Que X, Gillin FD: Cellular and transcriptional changes during excystation of Giardia lamblia in vitro. Exp Parasitol I998, 88: I 72 - I I 83.

20. Clement SL, Koslowsky DJ: Unusual organization of a developmentally regulated mitochondrial RNA polymerase (TBMTRNAP) gene in Trypanosoma brucei. Gene 2001, 272:209 $-2218$.

21. Felsenstein J: Cases in which parsimony and compatibility methods will be positively misleading. Syst Zool 1978, 27:40 I -4410 .

22. Peattie DA, Alonso RA, Hein A, Caulfield JP: Ultrastructural localization of giardins to the edges of disk microribbons of Giardia lamblia and the nucleotide and deduced protein sequence of alpha giardin. J Cell Biol 1989, 109:2323 -22335.

23. Pacold ME, Suire S, Perisic O, Lara-Gonzalez S, Davis CT, Walker EH, Hawkins PT, Stephens L, Eccleston JF, Williams RL: Crystal structure and functional analysis of Ras binding to its effector phosphoinositide 3-kinase gamma. Cell 2000, I 03:931-943.

24. Holberton DV, Marshall J: Analysis of consensus sequence patterns in Giardia cytoskeleton gene promoters. Nucleic Acids Res 1995, 23:2945 -22953.

25. Elmendorf HG, Singer SM, Nash TE: The abundance of sterile transcripts in Giardia lamblia. Nucleic Acids Res 200I, 29:4674 $-44683$.

26. Davies SP, Reddy H, Caivano M, Cohen P: Specificity and mechanism of action of some commonly used protein kinase inhibitors. Biochem J 2000, 35 I:95 - 105.

27. Yang P, Sale WS: Casein Kinase $I$ is anchored on axonemal doublet microtubules and regulates flagellar dynein phosphorylation and activity. J Biol Chem 2000, 275: I8905 -II89I2.

28. Kimura K, Hattori S, Kabuyama Y, Shizawa Y, Takayanagi J, Nakamura S, Toki S, Matsuda Y, Onodera K, Fukui Y: Neurite outgrowth of PCI 2 cells is suppressed by wortmannin, a specific inhibitor of phosphatidylinositol 3-kinase. I Biol Chem 1994, 269:|896|-|8967.

29. Woscholski R, Kodaki T, McKinnon M, Waterfield MD, Parker PJ: A comparison of demethoxyviridin and wortmannin as inhibitors of phosphatidylinositol 3-kinase. FEBS Lett 1994, 342:109-114.

30. Walker EH, Pacold ME, Perisic O, Stephens L, Hawkins PT, Wymann MP, Williams RL: Structural determinants of phosphoinositide 3-kinase inhibition by wortmannin, LY294002, quercetin myricetin, and staurosporine. Mol Cell 2000, 6:909-919.

31. Doerig C, Meijer L, Mottram JC: Protein kinases as drug targets in parasitic protozoa. Trends Parasitol 2002, I 8:366-37I.

32. He D, Wen JF, Chen WQ, Lu SQ, Xin de D: Identification, characteristic and phylogenetic analysis of type II DNA topoisomerase gene in Giardia lamblia. Cell Res 2005, I 5:474-4482.

33. Ward SG, Finan P: Isoform-specific phosphoinositide 3-kinase inhibitors as therapeutic agents. Curr Opin Pharmacol 2003, 3:426-434

34. Altschul SF, Madden TL, Schaffer AA, Zhang J, Zhang Z, Miller W, Lipman DJ: Gapped BLAST and PSI-BLAST: a new generation of protein database search programs. Nucleic Acids Res 1997, 25:3389 -33402

35. Schultz J, Milpetz F, Bork P, Ponting CP: SMART, a simple modu lar architecture research tool: identification of signaling domains. Proc Natl Acad Sci 1998, 95:5857 -55864.

36. Murzin AG, Brenner SE, Hubbar TJP, Chothia C: SCOP: A struc tural classification of proteins database for the investigation of sequences and structures. J Mol Biol 1995, 247:536 -5540.

37. Thompson JD, Higgins DG, Gibson TJ: CLUSTAL W: improving the sensitivity of progressive multiple sequence alignment through sequence weighting, position-specific gap penalties and weight matrix choice. Nucleic Acids Res 1994, 22:4673 -44680 .

38. McGuffin LJ, Bryson K, Jones DT: The PSIPRED protein struc ture prediction server. Bioinformatics 2000, 16:404 -4405.

39. Huelsenbeck JP, Ronquist F: MRBAYES: Bayesian inference of phylogenetic trees. Bioinformatics 200I, 17:754 -7755.

40. Guindon S, Gascuel O: A simple, fast, and accurate algorithm to estimate large phylogenies by maximum likelihood. Syst Biol 2003, 52:696-6704.
4I. Clark CG, Diamond LS: Methods for Cultivation of Luminal Parasitic Protists of Clinical Importance. Clin Microbiol Rev 2002, I 5:329 -334I.

42. Kane AV, Ward HD, Keusch GT, Pereira ME: In vitro encystation of Giardia lamblia: large-scale production of in vitro cysts and strain and clone differences in encystation efficiency. Parasitol |99|, 77:974 -998I.

43. Nixon JE, Wang A, Morrison HG, McArthur AG, Sogin ML, Loftus BJ, Samuelson J: A spliceosomal intron in Giardia lamblia. Proc Nat Acad Sci 2002, 99:370I -33705.

44. Hall TA: BioEdit: a user-friendly biological sequence alignment editor and analysis program for Windows 95/98/NT. Nucl Acids Symp Ser 1999, 41:95 -998.

45. Schwartz RM, Dayhoff MO: Origins of prokaryotes, eukaryotes, mitochondria, and chloroplasts. Science 1978, I99:395-403.

46. Lalle M, Salzano AM, Crescenzi M, Pozio E: The Giardia duodenalis 14-3-3 protein is post-translationally modified by phosphorylation and polyglycylation of the C-terminal tail. J Biol Chem 2006, 28I:5।37 -55 I48.

47. Morrison HG, Zamora G, Campbell RK, Sogin ML: Inferring protein function from genomic sequence: Giardia lamblia expresses a phosphatidylinositol kinase-related kinase similar to yeast and mammalian TOR. Comp Biochem Physiol B Biochem Mol Biol 2002, I 33:477-49I.
Publish with Biomed Central and every scientist can read your work free of charge

"BioMed Central will be the most significant development for disseminating the results of biomedical research in our lifetime. "

Sir Paul Nurse, Cancer Research UK

Your research papers will be:

- available free of charge to the entire biomedical community

- peer reviewed and published immediately upon acceptance

- cited in PubMed and archived on PubMed Central

- yours - you keep the copyright

Submit your manuscript here:

http://www.biomedcentral.com/info/publishing_adv.asp
BiolMedcentral 